Hier können Sie CME-Punkte sammeln a) für die Pflichtfortbildung aller Vertragsärzte und b) für freiwillige Fortbildungszertifikate, die viele Landesärztekammern anbieten.

Die Multiple-Choice-Fragen beziehen sich auf den vorangegangenen Fortbildungsbeitrag (Seiten 42-45). Die Antworten ergeben sich z. T. aus dem Text, z. T. beruhen sie auf medizinischem Basiswissen!

Wenn Sie 70\% der Fragen richtig beantworten, erhalten Sie 2, bei 100\% 3 CME-Punkte.

Teilnahmeschluss: 12. Oktober 2008 (www.cme-punkt.de) bzw. 26. Juni 2008 (Einsendung per Brief).
CME-Herausgeber- und Review-Board: Dr. H. J. K. Barwitz, Prof. Dr. A. Berghaus, Prof. Dr. Dr. h.c. Th. Brandt, Prof. Dr. W. G. Daniel, Prof. Dr. W. Eisenmenger, Prof. Dr. K. Friese, Prof. Dr. H. S. Füessl, Prof. Dr. B. Göke, Prof. Dr. R. Gradinger, Prof. Dr. H. Holzgreve, Prof. Dr. A. Imdahl, Prof. Dr. K.-W. Jauch, Prof. Dr. K. Krüger, Prof. Dr. H.-J. Möller, Prof. Dr. D. Reinhardt, Prof. Dr. Dr. h.c. Th. Ruzicka, Prof. Dr. Ch. Stief, U. Weigeldt.

\title{
Fragen zum Thema „Freie Flüssigkeit im Bauchraum“
}

- 1. Sie werden abends zu einem Patienten gerufen, der am selben Tag eine Leberblindpunktion erhalten hat und nun über rechtsseitigen Oberbauchschmerz klagt. Um zu überprüfen, ob sich freie Flüssigkeit im Morison-Pouch befindet, stellen Sie welche der folgenden Standardschnittebenen ein?

A Unterbauchquerschnitt

B Unterbauchlängsschnitt

C Flankenschnitt links

D Subkostaler Schrägschnitt links

E Paramedianer Längsschnitt bzw. Interkostalschnitt rechts

- 2. Welche der folgenden Konstellationen erfordert keine weitere Abklärung?

A Geringe Menge zyklusabhängiger freier Flüssigkeit bei der Frau

B Freie Flüssigkeit bei klinischem V.a. Leberzirrhose

C Freie Flüssigkeit bei klinischem V.a. Appendizitis

D Freie Flüssigkeit mit Leberraumforderung

E Freie Flüssigkeit mit unterer Einflussstauung

- 3. Eine 42-jährige Patientin stellt sich mit akuter Zunahme des Bauchumfangs und Oberbauchschmerz vor. Die klinische Untersuchung zeigt deutlichen Aszites. Sie vermuten angesichts einer bekannten Thrombophilie ein Budd-Chiari-Syndrom. Welcher sonografische Befund passt am ehesten dazu?

A Erweiterung der A. hepatica auf $>7 \mathrm{~mm}$

B Erweiterung der Lebervenen auf $>10 \mathrm{~mm}$

C Hyperechogene Leberraumforderungen

D Erweiterte intrahepatische Gallenwege mit Aerobilie

E Hepatomegalie, Hypertrophie des Lobus caudatus, Thrombose der Vv. hepaticae oder der V. cava

\section{-4. Als sicheres sonografisches Zeichen} einer portalen Hypertension gilt

A Dilatation der V. portae auf $>10 \mathrm{~mm}$.

B Rekanalisierung der Umbilikalvene.

C Splenomegalie.

D Gallenblasenwandverdickung

E Lymphknotenvergrößerung im Ligamentum hepatoduodenale.

- 5. Eine 67-jährige Patientin, die Sie seit Längerem wegen Steatosis hepatis behandeln, zeigt bei der abdominalen Sonografie neu aufgetretene freie intraperitoneale Flüssigkeit in Kombination mit mehreren Leberraumforderungen. Welche der folgenden Diagnosen ist am unwahrscheinlichsten?

A Hepatozelluläres Karzinom

B Lebermetastasen eines Mammakarzinoms

C Hepatische und peritoneale Metastasierung eines Kolonkarzinoms

D Leberzirrhose mit Regeneratknoten

E Fokale noduläre Hyperplasie

6. Welche Aussage zur sonografisch gesteuerten Aszitespunktion ist richtig?

A Sie ermöglicht eine exakte Diagnose und ist daher bei ausreichend breitem Aszitessaum immer indiziert.

B Das Risiko eines Hämoperitoneums ist bei manifester Leberzirrhose erheblich.

C Bei Leberzirrhose ist präinterventionell regelmäßig eine Substitution der Blutgerinnung erforderlich.

D Eine orientierende Farbdopplersonografie bringt keinen Informationsgewinn.

E Der Punktionsort ist mit dem bei Leberstanzbiopsie identisch.
- 7. Welche Aussage zur visuellen Beurteilung eines Aszites (nach Punktion) ist richtig?

A Eine Unterscheidung zwischen Leberzirrhose und Rechtsherzinsuffizienz ist anhand des Aszites problemlos möglich.

B Wenn der Patient fieberfrei ist, muss auch bei trübem Punktat nicht an eine spontan bakterielle Peritonitis gedacht werden.

C Rötlich verfärbter Aszites (i.e. > 10000

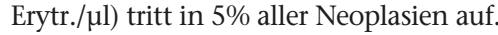

D Rötlich verfärbter Aszites (i.e. > 10000 Erytr./ $\mu$ l) tritt in 50\% aller hepatozellulären Karzinome auf.

E Rötlich verfärbter Aszites (i.e. > 10000 Erytr./ $\mu \mathrm{l})$ tritt in 100\% aller hepatozellulären Karzinome auf.

- 8. Ab welcher Konzentration polymorphkerniger Granulozyten spricht man von einem infizierten Aszites?
A $>50 / \mu \mathrm{l}$
B $>150 / \mu \mathrm{l}$
C $>250 / \mu 1$
D $>400 / \mu l$
E $>500 / \mu \mathrm{l}$

- 9. Die Berechnung des Serum-Aszites-Albumin-Gradienten (SAAG) ermöglicht eine gute Vorhersage einer portalen Hypertension. Grenzwert ist hier ein SAAG von
A $>0,1 \mathrm{~g} / \mathrm{dl}$
B $>0,5 \mathrm{~g} / \mathrm{dl}$
C $>1,0 \mathrm{~g} / \mathrm{dl}$
D $>1,1 \mathrm{~g} / \mathrm{dl}$
E $>2,1 \mathrm{~g} / \mathrm{dl}$.

- 10. Wie stellt sich unkomplizierter Aszites bei Rechtsherzinsuffizienz in der B-Bild-Sonografie typischerweise dar?

A Echofrei mit dilatierter V. cava

B Echofrei mit kollabierter V. cava

C Echofrei mit peritoneal fixierten Dünndarmschlingen

D Echofrei mit Leberraumforderung

E Echoreich

\section{ANTWORTFORMULAR}

Mit Einreichen dieses Fragebogen erkläre ich mich damit einverstanden, dass die angegebenen Daten zum Zweck der Teilnahmebestätigung gespeichert und bei erfolgreicher Teilnahme an den Einheitlichen Informationsverteiler (EIV) der Ärztekammern weitergegeben werden.

Wichtiger Hinweis:

Eine Auswertung ist künftig nur noch möglich, wenn Sie Ihre EFN auf dem Antwortformular angeben!

Nähere Hinweise hierzu unter: www.cme-punkt.de/faq.htm
„Freie Flüssigkeit im Bauchraum“

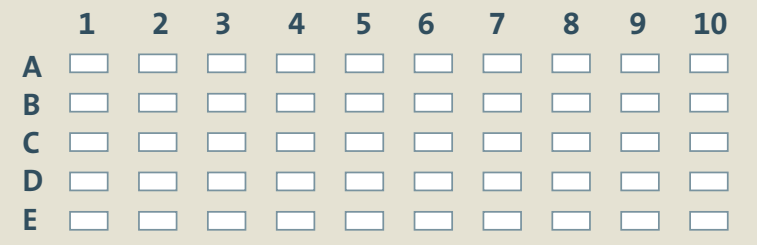

Bitte kreuzen Sie pro Frage je eine Antwort deutlich an.
Und so kommen Sie zu Ihren Punkten:

Teilnahme im Internet: unter www.cme-punkt.de. Dort führen wir für Sie ein elektronisches Punktekonto.

Teilnahme per Brief: Fragebogen ausfüllen und mit frankiertem Rückumschlag an Urban \& Vogel GmbH CME MMW-Fortschr. Med. Postfach 81664 München 